\title{
Greinke, Lena (2020): Berufsbedingte Multilokalität in ländlichen Räumen Niedersachsens. Gesellschaftliche und räumliche Auswirkungen als planerische Herausforderung am Beispiel des Landkreises Diepholz
}

\author{
Caroline Kramer \\ Eingegangen: 28. Februar 2021 - Angenommen: 29. März 2021 - Online veröffentlicht: 23. April 2021
}

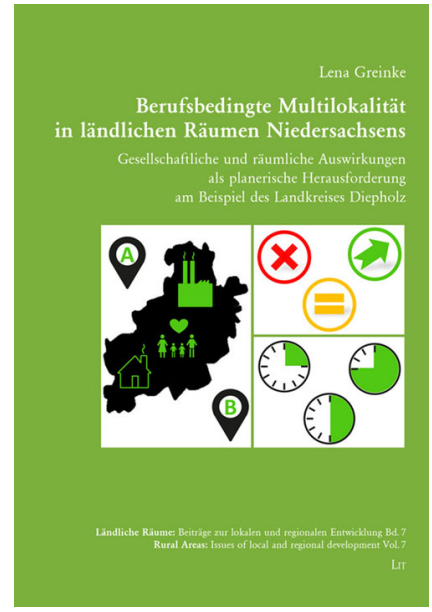

Das Thema Multilokalität ist in den letzten beiden Jahrzehnten sowohl in der Geographie als auch in den Sozialund Planungswissenschaften in den Fokus der Mobilitäts-, Migrations-, Stadt-, Familien- und Lebensstilforschung gerückt. Die berufsbedingte Multilokalität ist von besonderem Interesse, da sie als Ausdruck einer Arbeitswelt gilt, die den

Prof. Dr. Caroline Kramer, Institut für Geographie und Geoökologie, Karlsruher Institut für Technologie, Kaiserstraße 12, 76131 Karlsruhe, Deutschland caroline.kramer@kit.edu

\footnotetext{
(c) (1) (c) 2021 Kramer; licensee oekom verlag. This Open Access article is published under a Creative Commons Attribution 4.0 International License.
}

Menschen abverlangt, zunehmend flexibler und mobiler zu sein. Die Arbeitsplätze dieser - oft hochqualifizierten - Berufstätigen konzentrieren sich in Verdichtungsregionen und Großstädten. Ländliche Regionen dagegen werden oft als typische Quellregionen für Multilokale angesehen, in denen die Angehörigen oder Familien ,zurückbleiben' und an die das multilokale Haushaltsmitglied an seinen freien Tagen zurückkehrt.

Dieses Stereotyp stellt die Autorin Lena Greinke in ihrer Dissertation zum Thema der berufsbedingten Multilokalität in einer Region im ländlichen Niedersachsen infrage. Sie untersucht am Beispiel des Landkreises Diepholz, in dem einige große und international agierende Unternehmen angesiedelt sind, welche Ausprägungen multilokalen Lebens in den dortigen Unternehmen anzutreffen sind, das heißt, wie viele multilokale Zugezogene (incomings) dort arbeiten, wie sie ihren Alltag und ihre Wohnsituation gestalten und welche räumlichen und gesellschaftlichen Auswirkungen multilokales Leben in diesen ländlichen Regionen besitzt. Über diese subjektbezogene Perspektive hinaus nimmt sie auch die Unternehmen selbst und die betroffenen Kommunen in den Blick, um planerische Steuerungsmöglichkeiten für diese Region zu entwickeln.

Die Autorin leitet ihre Arbeit mit einem Kapitel zu den Forschungszielen ein und zeigt im nachfolgenden Kapitel zum Stand der Forschung, dass sie die einschlägige Literatur sowohl zum Thema Multilokalität in großer Breite als auch zu den Entwicklungsmöglichkeiten ländlicher Räume kennt. Dann erläutert sie fundiert die Auswahl ihrer Untersuchungsregion, ihre Untersuchungsmethoden und stellt den Untersuchungsraum im Detail vor. Ihren Forschungsfragen geht sie mit einem breiten Methodenmix nach: mit 
einer standardisierten Online-Befragung von Multilokalen in den Unternehmen und mit leitfadengestützten Interviews von Multilokalen, Vertreter/-innen der Unternehmen und der Kommunen. Zudem arbeitet sie mit der Methode der reflexiven Photographie, die von vertiefenden Gesprächen und weiteren Bewertungsmethoden begleitet wird. Ein wesentlicher Fokus ihrer Arbeit sind zwei Typologien, zum einen die der berufsbedingt multilokalen Incomings und zum anderen die der Unternehmen hinsichtlich ihres Umgangs mit multilokal lebenden Beschäftigten.

Nach den ersten hundert Seiten stellt Lena Greinke die eigenen empirischen Ergebnisse vor. Dies geschieht in einem ersten Schritt in Form einer deskriptiven Präsentation, die übersichtlich und graphisch ansprechend aufbereitet ist. Es zeigt sich, dass vor allem in den leitfadengestützten Interviews mit Multilokalen wichtige Aspekte des multilokalen Lebens angesprochen werden konnten, die mit gut ausgewählten Originalzitaten ,mit Leben gefüllt‘ werden können. Mit der Methode der reflexiven Photographie gelingt ein sehr lebendiger Einblick in die multilokale Lebenswelt an mehreren Orten. In Kombination mit Tabellen, in denen die Befragten verschiedene Aspekte der jeweiligen Orte bewerten können, entsteht eine interessante Mischung aus qualitativen und quantitativen Instrumenten. Es wird zum einen deutlich, wie unterschiedlich die Befragten mit dieser Herausforderung umgehen und welche Herausforderungen - vor allem bei der Wohnungssuche - im ländlichen Raum auf sie warten. Zum anderen werden bereits gewisse Muster sichtbar, die im Folgenden für die Typenbildung bedeutsam sind. Eine erste Analyse der Perspektive der Unternehmen und der der Funktionsträger/-innen zeigt, dass noch nicht bei allen Beteiligten angekommen ist, dass es diese besondere Gruppe der multilokal lebenden Beschäftigten in ihrer ländlichen Region gibt.

Die beiden Typologien von Multilokalen und von Unternehmen, die als ein zentrales Ziel der Arbeit angestrebt wurden, werden im vorletzten Kapitel der Arbeit entwickelt und vorgestellt. Zuvor wird eine Übersicht über ausgewählte, bereits bestehende Typologien gegeben, in der zum einen die Vielfalt dieser Typologien zu erkennen ist, gleichzeitig aber auch deren Lücken deutlich werden. Vor diesem Hintergrund entwickelt die Autorin ihre eigene Typologie in einem schrittweisen Prozess. Gut gelungen sind die Exkurse der sogenannten Personas, das heißt der fiktiven Beispiele, in denen Merkmale dieser Typen zusammengestellt werden. Es lassen sich für die Multilokalen drei Haupttypen erkennen, die sich im Wesentlichen hinsichtlich der Dauer ihrer multilokalen Lebensweise unterscheiden: ,kurzzeitige', ,mittelfristige“ und ,langfristige‘ Incomings, wobei sich unter den mittelfristigen Incomings ,ablehnende ' und ,akzeptierende' differenzieren lassen. In gleicher Weise wird die Typologie der Unternehmen entwickelt, die sich in ,Multilo-
kalität-Vermeidende“, ,Multilokalität-Neutrale ‘ und ,Multilokalität-Treibende' gliedert, wobei Letztere weiter in ,Unterstützende " und ,Fordernde " unterschieden werden.

Im letzten Kapitel werden die gesellschaftlichen und räumlichen Auswirkungen multilokaler Lebensweisen in dieser ländlichen Region ausführlich diskutiert, die Herausforderungen und Chancen für die Arbeitswelt und die Region thematisiert und Steuerungsmöglichkeiten von Seiten der Kommunen und der Akteure der Planung vor Ort und in der Region aufgezeigt. Es wird deutlich, dass sowohl in der Arbeitswelt als auch in der Lebenswelt Unterstützung möglich und wichtig wäre. Diese Unterstützung könnte von Homeoffice-Lösungen (die möglicherweise im vergangenen Jahr, nach den Untersuchungen von Lena Greinke, bereits realisiert wurden) über Unterstützung für Mobilität bis hin zur Versorgung mit spezifischen Wohnangeboten reichen. Insbesondere das im ländlichen Raum kaum vorhandene Angebot an kleineren (Miet)Wohnungen stellt eines der größten Probleme für Multilokale dar.

Im Ausblick werden Fragen aufgeworfen, die z. B. die Übertragbarkeit der Ergebnisse auf andere Regionen, den direkten Vergleich (groß)städtischer und ländlicher Räume oder aber die problematische Quantifizierung mithilfe von Melderegistern betreffen. Eines der wichtigsten Themen hat meines Erachtens durch die aktuelle Corona-Pandemie weiteren Nachdruck erhalten: Welche Auswirkungen wird die immer dynamischer voranschreitende Digitalisierung in der Arbeitswelt auf die multilokalen Lebens- und Arbeitswelten haben?

Die Arbeit von Lena Greinke ist eine lohnenswerte Lektüre nicht nur für Forschende im Feld Multilokalität, da sie die spezifische Situation multilokalen Arbeitens und Lebens in einer ländlichen Region beleuchtet. Sie ist auch für Unternehmen und Akteure in ländlichen Regionen interessant, da sie gut beraten sind, diese Gruppe wahrzunehmen und sich ihrer anzunehmen. Diejenigen, die sich einen raschen Überblick verschaffen möchten, können die tabellarischen Übersichten und die kurzen Kapitelzusammenfassungen nutzen, wobei Letztere noch hilfreicher wären, wenn man sie mithilfe der Gliederung auffinden könnte. Da zu vermuten ist, dass für Unternehmen und Akteure in Planung und Politik in ländlichen Räumen die vorliegende Arbeit eine zu umfangreiche Lektüre darstellt, wäre es meines Erachtens überlegenswert, aus dieser Arbeit eine kurze Handreichung für diese Zielgruppe zu entwickeln. 


\section{Vollständige bibliographische Angaben des}

\section{rezensierten Werkes:}

Greinke, Lena (2020): Berufsbedingte Multilokalität in ländlichen Räumen Niedersachsens. Gesellschaftliche und räumliche Auswirkungen als planerische Herausforderung am Beispiel des Landkreises Diepholz. Berlin: Lit Verlag. = Ländliche Räume. Beiträge zur lokalen und regionalen Entwicklung 7. 39 Tabellen, 36 Abbildungen, 257 Seiten 